\title{
Studying the influence of natural and artificial fracture on productivity of vertical well by hydroelectric simulation
}

\author{
Jian Yan ${ }^{1, ~ a, ~ M e i t a o ~ F u ~}{ }^{2, b}$, Xiaoyang Liü, \\ 1,2,3X'an Shiyou University, Xi'an, 710065, China \\ azhuimr99@163.com, b280781875@qq.com, ${ }^{c} 570137426 @$ qq.com
}

\begin{abstract}
Keywords: productivity of oil well; hydroelectric simulation; natural fracture; artificial fracture Abstract: Hydroelectric simulation is an important physical simulation method, providing a direct way to study the flowing law of fluid in reservoir. This paper carried out the hydroelectric simulation experiments to study the influence of growth of natural fracture, half-length and conductivity of artificial fracture on the productivity of oil well. The results indicate that: under the same permeability, with the increase of half-length of artificial fracture, the productivity of the oil well will increase, but when the length reaches a certain degree, the increasing degree will decrease, and the proper length of artificial fracture should be selected combining the numerical simulation results for the consideration of water cut; under the same permeability and half-length artificial fracture, the more the number of the natural fracture that link to the artificial fracture, the larger the productivity of the oil well; under the same reservoir condition and fracturing scale, the larger the conductivity of the artificial fracture, the higher the productivity of the oil well. The studying results provide theoretical references for studying the stimulation mechanisms of hydro fracturing, and checking the results of numerical simulation.
\end{abstract}

\section{Introduction}

Hydraulic fracturing in low permeability oil reservoir can change the flowing mode of fluid and stimulate the productivity of oil well. But the flowing law is complicated in reservoir, it is difficult to describe the flowing law accurately only by analytical and numerical simulation methods, so the physical simulation method is needed. The hydroelectric simulation is physical method according to the similarity principle of water and electricity field. It can reflect the flowing law directly and can check the accuracy of analytical and numerical simulation method [1,4]. This paper carried out the hydroelectric simulation experiments to study the influence of natural and artificial fracture on productivity of vertical well.

\section{Experimental principle and method}

\section{Experimental principle}

Based on the similarity of flowing mode between formation fluid and electric current, hydroelectric simulation simulates the flowing mode by physical experiment according to similar flowing formula. In the experiments, by injecting the solution with conductive ability, exerting a certain voltage on the boundaries of the container, the pressure field distribution of the formation fluid is simulated by the electric field distribution of solution. So the hydroelectric simulation can be used to simulate the stable flowing by the stable current.

Tab.1 Similarity principle of water and electric field (geometric similarity and physical similarity)

\begin{tabular}{lclc}
\hline \multicolumn{3}{c}{ Flowing field } & \multicolumn{2}{c}{ Electric field } \\
\hline Laplace formula & $\nabla{ }^{2} P=0$ & Laplace formula & $\nabla{ }^{2} U=0$ \\
Darcy law & $\nu=-K \bullet \nabla P / \mu$ & Ohm's law & $I=-\rho \nabla U$ \\
Flow rate & $v$ & Density of electric current & $J$ \\
Flow quantity & $q$ & Electric current & $I$ \\
Mobility & $K / \mu$ & Electric conductivity & $\rho$ \\
\hline
\end{tabular}




\section{Experimental equipment and fluids}

Experimental equipment is composed by four parts: reservoir simulation system, low voltage circuit system, spotting system and data collection system. The sodium chloride solution with a certain concentration and volume is used to simulate the reservoir medium; cell boundary is the closed boundary condition; copper strip is the supply boundary; small copper pipe is the wellbore. In the low voltage circuit system, the required voltage is provided by ac regulated power, and the voltage is output to the boundary and the wellbore.

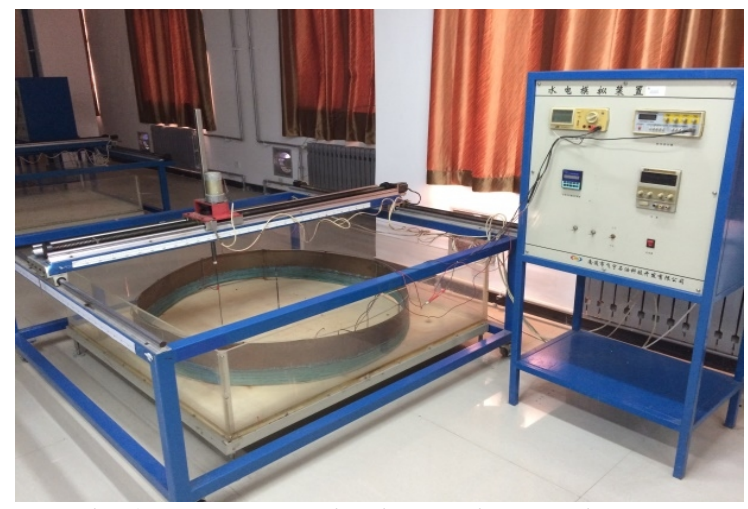

Fig.1 Hydroelectric simulation equipment

\section{Experimental methods}

According to the relationship between concentration of sodium chloride solution and conductivity, prepare corresponding sodium chloride solution to form a certain permeability reservoir, such as $0.5 \mathrm{mD}, 1.0 \mathrm{mD}, 10 \mathrm{mD}$ and so on;

Stick a certain length of copper sheet to the wellbore, forming a certain half-length $(0 \mathrm{~m}, 60 \mathrm{~m}$, $80 \mathrm{~m}, 100 \mathrm{~m}$, and $120 \mathrm{~m}$ ) artificial fracture, test the electric current (productivity of oil well), and analyze the influence of length of artificial fracture on productivity;

Distribute a certain number of copper wires on copper sheet to form natural fractures, test the electric current, and analyze the influence of growth of natural fracture on productivity;

Substitute the copper sheet with iron sheet, form the low conductivity artificial fracture, and test the electric current, and analyze the influence of conductivity of artificial fracture on productivity.

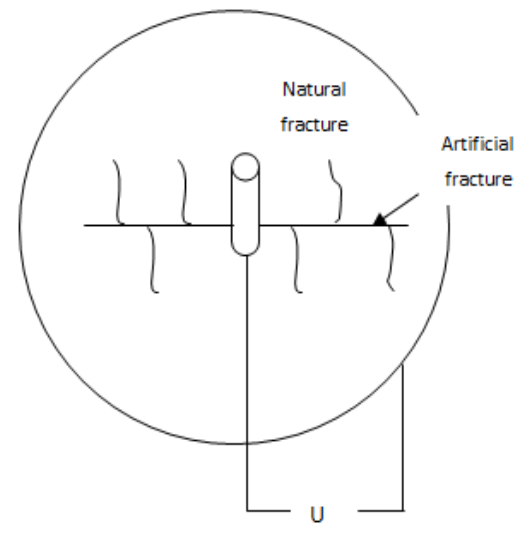

Fig. 2 Flow diagram for test the influence of natural fracture

\section{Analysis of experimental results}

\section{Influence of half-length of artificial fracture}

Figure 3 shows that: under the same permeability, with the increase of half-length of artificial fracture, the dimensionless productivity (current production rate divided by that without fracturing) of the oil well will increase, but when the length reaches a certain degree, the increasing degree of the 
productivity will decrease. That is, the longer the artificial fracture, the higher the productivity is, but not increasing all the time. And it also should note that: the productivity simulated is the liquid productivity, not the oil productivity; the experiment cannot simulate the change of water cut. So the water breakthrough caused by too long fracture in waterflooding oilfield should also be considered, the effect of which can be simulated by numerical simulation. Combining the numerical simulation results, the proper length of artificial fracture can be selected, which not only can ensure the small increasing speed of water cut, but also can keep a high productivity of oil.

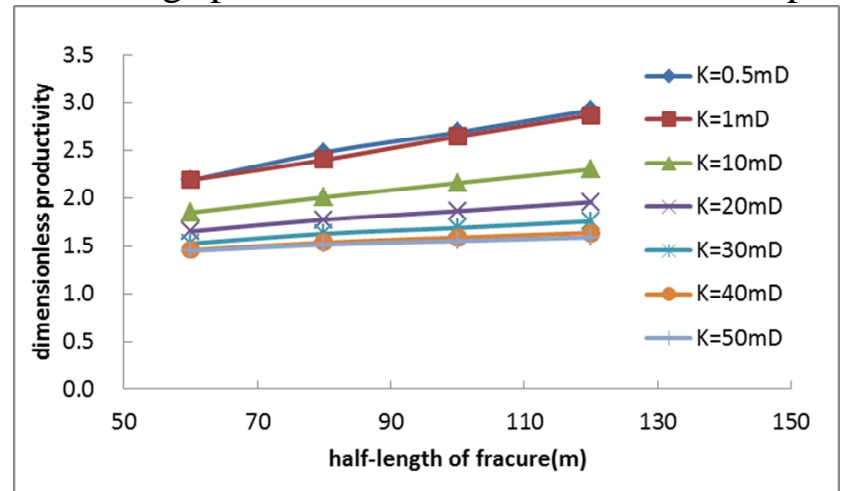

Fig.3 Effect of half-length of fracture on productivity

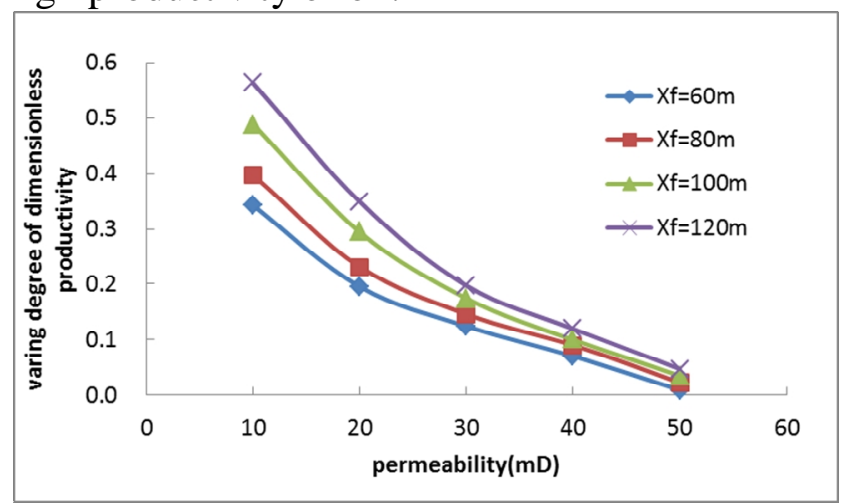

Fig. 4 Varing degree of dimensionless productivity vs permeability under different length of fracture

From figure 4, it can also be seen that under the same half-length of artificial fracture, with the increase of the permeability, the varing degree of the productivity is becoming small, that is, for the high permeability reservoirs, the stimulation effect is not obvoius than that of low permeability ones. If we want to get better stimulation effect, the longer half-length of artificial fracture is needed, but which may cause the fast rising speed of water cut.

\section{Influence of growth of natural fracture}

The results is shown in figure 5, it indicates that: under the same permeability and half-length artificial fracture, the more the number of the natural fracture that link to the artificial fracture, the larger the productivity of the oil well. That is, the more grown of the natural fracture in the reservoir, the more chance that artificial fracture can link with natural ones, so the productivity of the oil well will be larger. But when the number of natural fracture reaches to a certain value, the increasing degree of the productivity will decreases. The reason is that, when the number of the natural fracture increases, the touching area of fracture with the reservoir will be larger, so the drainage area and the productivity will increases. However, from the principle of potential superposition, it is known that with the increase of density of the natural fracture, the mutual effect among the fractures will become stronger, so the increasing degree of productivity will be limited.

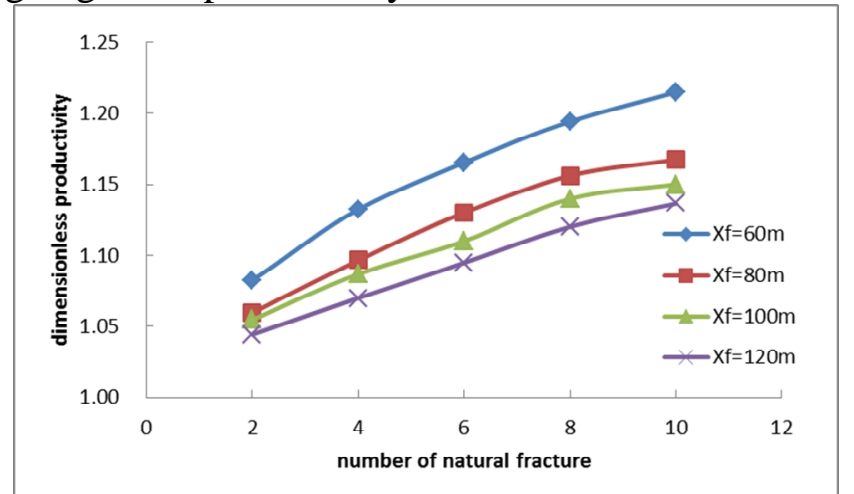

Fig. 5 Effect of growth of natural fracture on productivity

\section{Influence of fracture conductivity}

From the experimental results (Fig.6), it can be seen that under the same permeability, the productivities tested by copper sheet are all larger than that tested by iron sheet. As it is known that the resistance of iron sheet is larger than that of copper, so the fracture simulated by iron sheet is the relative low conductivity fracture, while the fracture simulated by copper sheet is the high 
conductivity fracture. That means, under the same reservoir condition and fracturing scale, the larger the conductivity of the artificial fracture, the higher the productivity of the oil well. This is why refracturing is conducted in oil field when the conductivity of fracture is becoming low which results the decreasing productivity. By refracturing, the conductivity of the fracture is recovered, and the productivity of the well will be improved. This is also why the high conductivity fracture should be formed in the process of hydraulic fracturing, which results high productivity oil well. The figure 7 also shows that with the increase of the half-length of artificial fracture, the increasing degree of productivity is becoming weak; the larger the permeability of the reservoir is, the weaker the effect of conductivity on fracturing well, that is, the stimulation effect is more obvious in low permeability reservoirs.

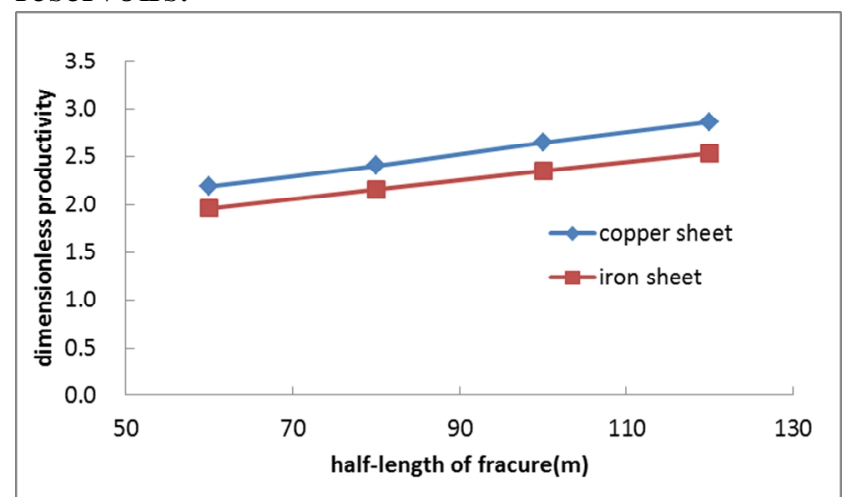

Fig.6 Effect of conductivity on productivity $(K=1.0 \mathrm{mD})$

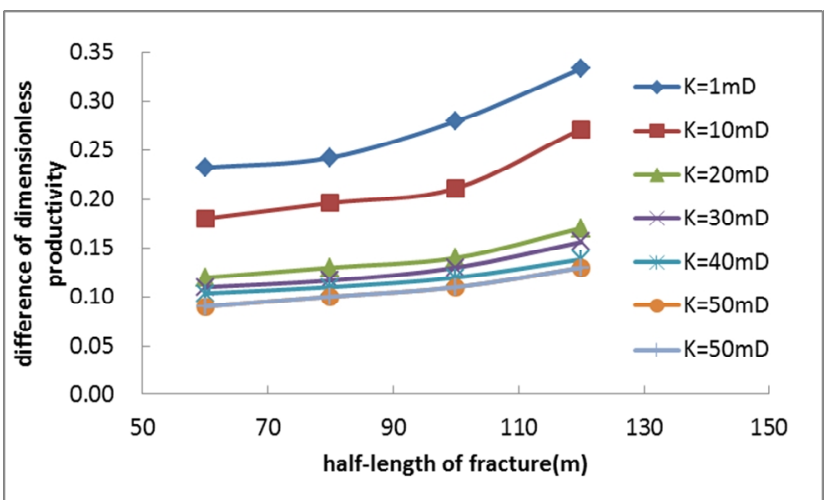

Fig. 7 Difference of dimensionless productivity tested under different conductivity fracture

\section{Conclusion}

Through the experiment of hydroelectric simulation, it can be known that:

Under the same permeability, with the increase of half-length of artificial fracture, the productivity of the oil well will increase, but when the length reaches a certain degree, the increasing degree will decrease, and the proper length of artificial fracture should be selected combining the numerical simulation results for the consideration of water cut;

Under the same permeability and half-length artificial fracture, the more the number of the natural fracture that link to the artificial fracture, the larger the productivity of the oil well;

Under the same reservoir condition and fracturing scale, the larger the conductivity of the artificial fracture, the higher the productivity of the oil well.

\section{Acknowledgments}

We would like to thank College of Petroleum Engineering in Xi'an Shiyou University for providing experimental conditions. In addition, it is supported by the national natural science fund item "Influence mechanism and correction of gas \& water relative permeability curve in tight gas reservoir" (51404197).

\section{References}

[1] GAO Fei, HAN Guo-qing etc. The Research of Experimental Method about Hydroelectric Simulation[J]. Science Technology and Engineering. Volume 12, Issue 16, 2012:3839-3843

[2] ZENG Baoquan CHENG Linsong etc. Development evaluation of fractured horizontal wells in ultra-low permeability reservoirs[J]. ACTA PETROLEI SINICA. Volume 31, Issue 5, 2010:791-796

[3] LI Chun-lan, CHENG Lin-song etc. Application of Wheatstone bridge in hydroelectric simulation experiment[J]. Laboratory Science. Volume 14, Issue 3, 2011:96-98

[4] Yao Ze. Electrolytic simulation experiment study on fractured development of horizontal wells in naturally reservoir[J]. Reservoir Evaluation and Development. Volume 2, Issue 1, 2013:22-26 